\title{
Reviewer acknowledgement
}

\author{
Sue T Griffin ${ }^{1}$ and Robert E Mrak ${ }^{2 *}$
}

\section{Contributing reviewers}

The editors of Journal of Neuroinflammation would like to thank all our reviewers who have contributed to the journal in Volume 12 (2015).

Lailla Abdullah

United States of America

Rosella Abeti

United Kingdom

Yousef Abu-Amer

United States of America

Dong Ahn

Republic of Korea

Saba Aïd

France

Yousef Al-Abed

United States of America

Philipp Albrecht

Germany

Jessy Alexander

United States of America

Lena Al-Harthi

United States of America

Artino Allen

United States of America

Joshua Allen

United States of America

Lydia Alvarez

Spain

Diana Amantea

Italy
Fumimasa Amaya

Japan

Winfried Amoaku

United Kingdom

Aileen Anderson

Lebanon

Anuska Andjelkovic Zochowska

United States of America

Zane Andrews

Australia

Juan Anguita

Spain

Daniel Anthony

United Kingdom

Ichio Aoki

Japan

Revital Aricha

Israel

Kiyoshi Ariizumi

United States of America

Regina Armstrong

United States of America

Rand Askalan

Canada

Stéphane Auvin

France
Hafidi Aziz

France

Ana Baamonde

Spain

Adam Bachstetter

United States of America

Ryan Bachtell

United States of America

Veerle Baekelandt

Belgium

Fulvio Baggi

Italy

David Baker

United Kingdom

Silvia Balosso

Italy

Giorgos Bamias

Greece

Banani Banerjee

United States of America

William Banks

United States of America

Steven Barger

United States of America

Anne Baron-Van Evercooren

France

\footnotetext{
* Correspondence: MrakRobertE@uams.edu

${ }^{2}$ Department of Pathology, University of Toledo Health Sciences Campus,

Toledo, USA

Full list of author information is available at the end of the article
} 
Ruth Barrientos

United States of America

Anirban Basu

India

Jan Bauer

Austria

Ingo Bechmann

Germany

Andreas Beineke

Germany

Jean-Pierre Bellier

Japan

Kendra Bence

United States of America

Jeffrey Bennett

United States of America

Thomas Berger

Austria

Cornelia Bergmann

United States of America

Kristen Bernard

United States of America

Sonia Berrih-Aknin

France

Sonja Best

United States of America

Knut Biber

Germany

Roger Bick

United States of America

Paula Bickford

United States of America

Helle Bielefeldt-Ohmann

Australia

Stacy Bilbo

United States of America

Silvia Bisti

Italy

Gregory Bix

United States of America

Gregory Bix

United States of America

Klas Blomgren

Sweden
Mathew Blurton-Jones

United States of America

Juan Bolanos

Spain

Kathryn Bollinger

United States of America

Jessica Bolton

United States of America

Bruno Bonaz

France

Karin Borges

Australia

Cesar Borlongan

United States of America

Kenneth Bost

United States of America

Charles Bradberry

United States of America

Monika Bradl

Austria

Lars-Ove Brandenburg

Germany

David Braun

United States of America

Amy Brewster

United States of America

Kris Brickman

United States of America

Celia Brosnan

United States of America

Brad Broughton

Australia

Jonathan Brouillette

Canada

Guy Brown

United Kingdom

Joan Brown

United States of America

Wolfgang Brück

Germany

Luigi Bubacco

Italy

Marion Buckwalter

United States of America
Bruce Bunnell

United States of America

Irina Burd

United States of America

Ta Butterick

United States of America

Kimberly Byrnes

United States of America

Huaibin Cai

United States of America

Lilian Calderon-Garciduenas

United States of America

Patrick Calders

Belgium

Ruth Caldwell

United States of America

Sean Callanan

Ireland

Arezoo Campbell

United States of America

Matthew Campbell

Ireland

Eduardo Candelario-Jalil

United States of America

Ling Cao

United States of America

Jeffrey Capadona

United States of America

Daniel Carr

United States of America

Richard Carr

Germany

Monica Carson

United States of America

Anna Carta

Italy

Ignazio Castagliuolo

Italy

Bogdan Catalin

Germany

Paola Cavalcante

Italy

Paramita Chakrabarty

United States of America 
Saikat Chakraborty

United States of America

Angel Chamorro

Spain

Andrew Chan

Germany

Alexandre Chan

Singapore

Gusharan Chana

Australia

Christiane Charriaut-Marlangue

France

Abhijit Chaudhuri

United Kingdom

Maxim Cheeran

United States of America

Sylvain Chemtob

Canada

Chu Chen

United States of America

Szu-Fu Chen

Taiwan Republic of China

Chun-Jung Chen

Taiwan Republic of China

Zhihong Chen

United States of America

Jonathan Cherry

United States of America

Glyn Chidlow

Australia

Isaac Chiu

United States of America

Sunghee Cho

United States of America

Sang-Ho Choi

United States of America

Michael Chopp

United States of America

Kon Chu

Republic of Korea

J. Clark

United States of America

Diego Clemente

Spain
Manola Comar

Italy

Colin Combs

United States of America

Phillippe Corcia

France

Peter Crack

Australia

David Cribbs

United States of America

Anne Cross

United States of America

E Cudaback

United States of America

Colm Cunningham

Ireland

Riccardo Cuppini

Italy

Daniela Curti

Italy

Krzysztof Czaja

United States of America

Endre Czeiter

Hungary

Cleide Da Silva

United States of America

Haibin Dai

China

Russell Dale

Australia

Robert Dantzer

United States of America

Kunjan Dave

United States of America

Terry Davidson

United States of America

Randall Davis

United States of America

Alejandro De Denicola

Argentina

Pierre De Graan

Netherlands

Joris De Man

Belgium
Rocío M. De Pablos

Spain

Tara De Silva

United States of America

Terrence Deak

United States of America

Rashid Deane

United States of America

Gregory Dekaban

Canada

Sharon Demorrow

United States of America

Gerald V. Denis

United States of America

Candan Depboylu

Germany

Matthew Derrick

United States of America

Ronald Deumens

Belgium

Kumlesh Dev

Ireland

Carlie Devries

Netherlands

Courtney Devries

United States of America

Cameron Dezfulian

United States of America

Barbara Di Benedetto

Germany

Betty Diamond

United States of America

Exuperio Diez-Tejedor

Spain

Christine D. Dijkstra

Netherlands

Charles Dinarello

United States of America

Kelley Dineley

United States of America

Yuchuan Ding

United States of America

Rosario Donato

Italy 
Catherine Dostert

Luxembourg

Patrick Dougherty

United States of America

Sarah Doyle

Ireland

Alex Dregan

United Kingdom

Paul Drew

United States of America

Kirk Druey

United States of America

Ronald Duman

United States of America

Aaron Dumont

United States of America

Alexander Easton

Canada

David Edwards

United States of America

Arik Eisenkraft

Israel

Joseph El Khoury

United States of America

Najira El-Hage

United States of America

Ll Elias

Brazil

Michal Elovitz

United States of America

Azza El-Remessy

United States of America

Mitsuhiro Enomoto

Japan

Adviye Ergul

United States of America

Michelle Erickson

United States of America

Ana Esquifino

Spain

Elsa Fabbretti

Slovenia

Sarah Falk

Denmark
Brian Fallon

United States of America

Chen-Ming Fan

United States of America

Cinthia Farina

Italy

Krysten Farjo

United States of America

Geraldine Favrais

France

Douglas Feinstein

United States of America

Robert Felder

United States of America

Vicente Felipo

Spain

Hua Feng

China

Patricia Fernandez

Panama

Javier Fernandez-Ruiz

Spain

Elisabeth Fernell

Sweden

Donna Ferriero

United States of America

Bernd Fiebich

Germany

Stefano Fiorucci

Italy

Mark Fisher

United States of America

Loretta Flanagan-Cato

United States of America

Mary Ann Fletcher

United States of America

Willem-Jan Fokkink

Netherlands

Laura Fonken

United States of America

G Forloni

Italy

Carola Forster

Germany
Thomas C. Foster

United States of America

Fornai Francesco

Italy

Joseph Francis

United States of America

Martin Frasch

Canada

Sally Frautschy

United States of America

Gregory Freund

United States of America

Manuel Friese

Germany

John Fryer

United States of America

Wen-Mei Fu

Taiwan Republic of China

Dietmar Fuchs

Austria

Ken-Ichiro Fukuchi

United States of America

Roberto Furlan

Italy

Prasad Gabbita

United States of America

Bhakta Prasad Gaire

Republic of Korea

Rob Galinsky

New Zealand

Lucio Gama

United States of America

Me Gambuzza

Italy

Olgo Garaschuck Germany

Lidia Garcia-Bonilla

United States of America

Borja García-Bueno

Spain

D Garcia-Ovejero

Spain

Gwenn Garden

United States of America 
Peter Garred

Denmark

Peter Gaskill

United States of America

Julie Gavard

France

Harris Gelbard

United States of America

John Gensel

United States of America

Steve M Gentleman

United Kingdom

Igal Gery

United States of America

Simantini Ghosh

United States of America

Othman Ghribi

United States of America

Francesca Gilli

United States of America

Pietro Giusti

Italy

Jonathan Godbout

United States of America

Norbert Goebels

Germany

Beryl Gok

United States of America

Ralf Gold

Germany

Yona Goldshmit

Israel

Diego Gomez-Nicola

United Kingdom

Michael Goodman

United States of America

Nicolai Gorbunov

United States of America

Carmen Gottfried

Brazil

Peter Grace

United States of America

Peter Grace

United States of America
Manuel Graeber

Australia

Elizabeth Gray

United Kingdom

Kim Green

United States of America

Paul Green

United States of America

Kim Green

United States of America

Elisa Greggio

Italy

Diane Griffin

United States of America

Christian Grimm

Switzerland

Wolfgang Grisold

Austria

Floris Groenendaal

Netherlands

Antje Grosche

Germany

Ross Gruber

United States of America

Alexandra Grubman

Australia

Franz Grus

Germany

Jean-Charles Guéry

France

Alistair Gunn

New Zealand

Rainer Haberberger

Australia

Abigail Hackam

United States of America

Marc Halterman

United States of America

Khalid Hanafy

United States of America

Andrew Harkin

Ireland

Ashley Harms

United States of America
Marni Harris-White

United States of America

Higashida Haruhiro

Japan

David Hasan

United States of America

Tomoki Hashimoto

United States of America

Johnny He

United States of America

Shuhan He

United States of America

Johnny He

United States of America

Raimund Helbok

Austria

Michael Heneka

Germany

Michael Hennessy

United States of America

Kenneth Hensley

United States of America

Marina Hernandes

United States of America

Paco Herson

United States of America

David Hess

United States of America

James Hewett

United States of America

Sandra Hewett

United States of America

Hubertus Himmerich

Germany

Rabea Hinkel

Germany

Holly Hinson

United States of America

Yoshitaka Hirooka

Japan

Silke Hirsch

Germany

Roger Ho

Singapore 
Shelley Hooks

United States of America

Douglas Hooper

United States of America

Kathryn Hopperton

Canada

Motohiro Horiuchi

Japan

Charles Howe

United States of America

Christine Hsieh

United States of America

Huijuan $\mathrm{Hu}$

United States of America

Xiaoming $\mathbf{H u}$

United States of America

Rui Huang

China

Christian Humpel

Austria

Fumito Ichinose

United States of America

O Igwe

United States of America

Hiroko Ikeshima-Kataoka

Japan

Jeffrey Iliff

United States of America

Atsuko Inoue

Japan

David Irani

United States of America

Takayuki Itoh

United States of America

Jason Ivanusic

Australia

Koichi Iwata

Japan

Joel Jackobsson

Sweden

Sanjay Jain

United States of America

Tatjana Jakobs

United States of America
Krzysztof Janeczko

Poland

Glen Jeffery

United Kingdom

Hui-Rong Jiang

United Kingdom

Meiyan Jiang

United States of America

Erik Johnson

United States of America

John Johnson

United States of America

Aaron Johnson

United States of America

Nicole Jones

Australia

Ilona Joniec-Maciejak

Poland

Chung Ju

Republic of Korea

Robert Kahoud

United States of America

Ryuji Kaji

Japan

Shuji Kaneko

Japan

Scott Kanoski

United States of America

A. Kanthasamy

United States of America

William Karpus

United States of America

Toshihiko Katafuchi

Japan

Yasufumi Kataoka

United States of America

Hiroshi Katsuki

Japan

Anu Kauppinen

Finland

Syed Kazim

United States of America

Richard Keep

United States of America
Samia Khoury

Lebanon

Mahmoud Kiaei

United States of America

Anton Kichev

United Kingdom

Ho Jin Kim

Republic of Korea

Jung-Ae Kim

Republic of Korea

Hyoung-Chun Kim

Republic of Korea

Myeung Ju Kim

Republic of Korea

Nicholas King

Australia

T Kiyota

United States of America

Christoph Kleinschnitz

Germany

Ingo Kleiter

Germany

Firas Kobeissy

United States of America

Jae Young Koh

Republic of Korea

Hideo Kohno

Japan

Ron Kooijman

Belgium

Juerfen Kopitz

Germany

Thomas Korn

Germany

S. Korte

Netherlands

Elöd Körtvely

Germany

Robert Kraft

Germany

Chia-Yi Kuan

United States of America

Markus Kuehn

United States of America 


\author{
Stefanie Kuerten \\ Germany \\ Patrick Kuery \\ Germany \\ Donald Kuhn \\ United States of America \\ Stephanie Kullmann \\ Germany \\ Anil Kumar \\ United States of America \\ Jinn-Rung Kuo \\ Taiwan Republic of China \\ Jacek Kurzepa \\ Poland
}

Katarzyna Kwiatkowska

Poland

Anne La Flamme

New Zealand

Jose L Labandeira-Garcia Spain

Steve Lacroix

Canada

Mary Jo. Ladu

United States of America

Philip Landfield

United States of America

Gary Landreth

United States of America

Thomas Lane

United States of America

Thomas Langmann

Germany

Mj Lavoie

United States of America

Catherine Lawrence

United Kingdom

David Lawrence

United States of America

Marcus Lawson

United States of America

Eric Lazartegues

United States of America

Eric Lazartigues

United States of America
John Lee

Australia

Sung Joong Lee

Republic of Korea

Sung Joong Lee

Republic of Korea

Sunhee Lee

United States of America

Sunhee Lee

United States of America

Christian Lefebvre D'hellencourt

France

Donald Lehmann

United Kingdom

Helmar Lehmann

Germany

Michael Lehmann

United States of America

Emily Leibovitch

United States of America

Guilhian Leipnitz

Brazil

Marcel Leist

Germany

Vincent Lelievre

France

Lisa Leon

United States of America

Christina Leslie

United States of America

Michael Levy

United States of America

Patrick Lewis

United Kingdom

Frank Leypoldt

Germany

Didier Leys

France

Cai Li

United States of America

J Li

United States of America

Junfa Li

China
Jian Liang

United States of America

Daniel Liebl

United States of America

Fabian Liechti

Switzerland

Jihyeon Lim

United States of America

Charles L. Limoli

United States of America

Raija Lindberg

Switzerland

Dan Lindholm

Finland

Eng-Ang Ling

Singapore

Ralf Linker

Germany

Gregory Liou

United States of America

Rob Lisak

United States of America

Lucia Lisi

Italy

Fudong Liu

United States of America

Lixin Liu

Canada

Nk Liu

United States of America

Jialing Liu

United States of America

Yawei Liu

Denmark

Yutong Liu

United States of America

Jordi Llop

Spain

Sarah Elisabeth Lloyd

United Kingdom

Eng Lo

United States of America

David Loane

United States of America 
David Loeffler

United States of America

James Lokensgard

United States of America

Ruben Lopez-Vales

Spain

Lisa Loram

United States of America

Seth Love

United Kingdom

Qingxian Lu

United States of America

Ru-Band Lu

Taiwan Republic of China

Paul Lucassen

Netherlands

Ralph Lucus

Germany

Jan Luenemann

Switzerland

Jia Luo

United States of America

Sarah Lutz

United States of America

Marina Lynch

Ireland

Daqing Ma

United Kingdom

Qingyi Ma

China

Halina Machelska

Germany

Andrew Maclean

United States of America

Akiko Maeda

United States of America

Sanjay Maggirwar

United States of America

Kathleen Maguire-Zeiss

United States of America

Pamela Maher

United States of America

Sabatino Maione

Italy
Marzia Malcangio

United Kingdom

Anne-Marie Malfait

United States of America

Carina Mallard

Sweden

Frederic Manfredsson

United States of America

Santhakumar Manicassamy

United States of America

Mark Mapstone

United States of America

Yannick Marchalant

United States of America

Nicola Marchi

United States of America

Carmela Marino

Italy

Daniel Marks

United States of America

Paola Marmiroli

Italy

Ian Marriott

United States of America

Thomas Martin

United States of America

J Martinez-Gonzalez

Spain

Ricardo Martinez-Zamudio

France

Rudolf Martini

Germany

Ángeles Martín-Requero

Spain

Eliezer Masliah

United States of America

Iara Massias-Reason

Brazil

Clayton Mathews

United States of America

Kaspar Matiasek

Germany

F Matsuda

Japan
Carlow Matute

Spain

Anne Kathrin Mausberg

Germany

Christian Mawrin

Germany

Audrey Mazarati

United States of America

Mervyn Maze

United States of America

Simon Mcarthur

United Kingdom

Louise Mccullough

United States of America

Robert Mccusker

United States of America

Braden Mcfarland

United States of America

James Mcginnis

United States of America

Declan Mckernan

Ireland

Gordon Meares

United States of America

Stephane Melik Parsadaniantz

France

Richard Mellanby

United Kingdom

Til Menge

Germany

Doron Merkler

Germany

Axel Methner

Germany

Jeffrey Meyer

Canada

Gerd Meyer Zu Horste

Germany

Geert Meyfroidt

Belgium

Jens Mikkelsen

Denmark

Djordje Miljkovic

Serbia And Montenegro 
C Miller

United States of America

Donald Miller

Canada

Dennis Miller

United States of America

Robert Miller

United States of America

Richard Miller

United States of America

Rachel Miller

United States of America

Richard Milner

United States of America

Luisa Minghetti

Italy

Yasushi Miura

Japan

Takuro Miyazaki

Japan

Ehsan Moazen Zadeh

Islamic Republic Of Iran

Alon Monsonego
Israel

Joan Montaner

Spain

Darren Moore

United States of America

James Morgan

United Kingdom

Dave Morgan

United States of America

Kouichi Morita

Japan

Melissa Moss

United States of America

Bruno Mourvillier

France

Elliott Mufson

United States of America

Angelika Mühlebner

Netherlands

Rob Mullins

United States of America
Benjamin Murdock

United States of America

Erland Nagelhus

Norway

Istvan Nagy

United Kingdom

Matthias Nahrendorf

United States of America

Ranuka Nair

India

Kunihiro Nakai
Japan
Hideaki Nakajima
Japan

Mike Namaka

Canada

Roland Nau

Germany

Christian Naus

Canada

Lucia Negri

Italy

Andrew Neilson

United States of America

Vivek Nerurkar

United States of America

Eric Ng

United States of America

Michael Nichols

United States of America

Robert Nickells

United States of America

Robert Nitsch

Germany

Christopher Norris

United States of America

Kelly Nudelman

United States of America

M. Kerry O'banion

United States of America

Birgit Obermeier

United States of America

Jason O'connor

United States of America
Janis O'donnell

United States of America

Halina Offner

United States of America

Toru Ogata

Japan

U Oh

United States of America

Andreas Ohlmann

Germany

K Ohno

Japan

Toshio Ohshima

Japan

Johanna Ojala

Finland

Tatsusada Okuno

Japan

John Olschowka

United States of America

Julie Olson

United States of America

Benjamin Ondruschka

Germany

Hiroaki Ooboshi

Japan

Lisa Opanashuk

United States of America

Gregory Ordway

United States of America

Michael Osthoff

Switzerland

Trevor Owens

Denmark

Yoko Ozawa

Japan

Rosana Pagano

Brazil

Guylene Page

France

Kalipada Pahan

United States of America

Maria Panaro

Italy 


\author{
Alberto Panerai \\ Italy \\ Francesco Parmeggiani \\ Italy
}

Rimma Parnova

Russian Federation

Guilio M. Pasinetti

United States of America

Cesare Patrone

Sweden

Gesine Paul

Sweden

Prasit Pavasant

Thailand

Krystyna Pawlak

Poland

Marcela Pekna

Sweden

Rosalia Pellitteri

Italy

Luis Penalva

United States of America

Yu-Ping Peng

China

Keith Pennypacker

United States of America

Liyanage Perera

United States of America

Karin Peterson

United States of America

A Petit-Paitel

France

Ashley Petrone

United States of America

Mary Petzke

United States of America

Mario Philipp

United States of America

Joanna Phillips

United States of America

Irene Pichler

Italy

Christian Pike

United States of America
Kelly Pike

Canada

Sudheesh Pilakka Kanthikeel

United States of America

Paulo Pires

United States of America

Anna Planas

Spain

Patrizia Pontisso

Italy

Dean Pountney

Australia

David Powell

United States of America

Elizabeth Powell

United States of America

Christopher Power

Canada

Domenico Pratico

United States of America

Darwin Prockop

United States of America

Barbara Przewlocka

Poland

Chaim Putterman

United States of America

Zenaide Quezado

United States of America

Richard Quigg

United States of America

Andre Quincozes-Santos

Brazil

P Rakoczy

Australia

Glenn Rall

United States of America

M. Ramanathan

India

Matt Ramer

United States of America

Ana Ramirez

Spain

G. Rebeck

United States of America
Raymond Regan

United States of America

William Regenold

United States of America

Theo Rein

Germany

Markus Reindl

Austria

Tonia Rex

United States of America

Susanna Ricci

Italy

Gianfranco Risuleo

Italy

Fatima Rivera

United States of America

Phillip Rivera

United States of America

Robert Roach

United States of America

Mayela Rodriguez

Mexico

Moses Rodriguez

United States of America

Mary-Louise Rogers

Australia

Gu Seob Roh

Republic of Korea

Troy Rohn

United States of America

Julian Romero

Spain

Lorenzo Romero-Ramirez Spain

M Romero-Ramos

Denmark

Christopher Rose

Canada

Anna Rosell

Spain

Thad Rosenberger

United States of America

Susanna Rosi

United States of America 
Kharah Ross

United States of America

Joachim Roth

Germany

Steven Roth

United States of America

Guido Rubboli

Denmark

Marc Ruitenberg

Australia

Christoph Rummel

Germany

Klemens Ruprecht

Germany

Horea Rus

United States of America

Karsten Ruscher

Sweden

Jong Ryu

Republic of Korea

Daniel Saban

United States of America

Roger Sabbadini

United States of America

Ahmad Salehi

United States of America

Samina Salim

United States of America

Paul Salvaterra

United States of America

Tarek Samad

United States of America

Vanesa Sanchez-Guajardo

Denmark

Juergen Sandkuehler

Austria

Lauren Sansing

United States of America

Abel Santamaria

Mexico

Ana Santiago

Portugal

Michael Santillo

United States of America
Thomas Santoro

United States of America

Rebecca Sappington

United States of America

Manuel Sarasa

Spain

Sohobhan Sarkar

Canada

Harvey Sarnat

Canada

Norman Saunders

Australia

Josep Saura

Spain

Valentina Savchenko

United States of America

Cristoforo Scavone

Brazil

Eliana Scemes

United States of America

Tanja Schlereth

Germany

Jürgen Schneider- Schaulies

Germany

Herbert Schwarz

Singapore

Guillaume Sebire

Canada

Jose Segovia

Mexico

Fatima Sehba

United States of America

Miho Sekiguchi

Japan

Dana Selley

United States of America

Bridgette Semple

Australia

Mrudang Shah

United States of America

Zahoor A. Shah

United States of America

Lee Shapiro

United States of America
Hari Sharma

Sweden

Hayley Shawn

Canada

Kazim Sheikh

United States of America

Jiangang Shen

China

John Sheridan

United States of America

T Shin

Republic of Korea

Seiji Shioda

Japan

Esther Shohami

Israel

Veronica Shubayev

United States of America

Rafael Simo

Spain

Roger Simon

United States of America

Dario Siniscalco

Italy

Luigi Sironi

Italy

Stephen Skaper

Italy

Thomas Skripuletz

Germany

Ann Smith

United States of America

Jessica Snowden

United States of America

Tomas Sobrino

Spain

Betty Soliven

United States of America

Volkan Solmaz

Turkey

Claudia Sommer

Germany

Yoshifumi Sonobe

Japan 
Linda Sorkin

United States of America

Lydia Sorokin

Germany

Rosa Sorrentino

Italy

Lirlandia Sousa

Brazil

Peter Spaeth

Switzerland

\section{James St John}

Australia

Philip Stahel

United States of America

Martin Stangel

Germany

Larry Steinman

United States of America

Mark Stettner

Germany

Jonathan Stiles

United States of America

Jonathan Stone

Australia

T Stone

United Kingdom

Barbara Stonestreet

United States of America

Wolfgang J. Streit

United States of America

Tatyana Strekalova

Netherlands

Stephen Strom

Sweden

Hyeon-Sook Suh

United States of America

Hyeon-Sook Suh

United States of America

Kyoungho Suk

Republic of Korea

Grace Sun

United States of America

Stevens Susan

United States of America
Tobias Suter

Switzerland

Raymond Swanson

United States of America

Fabian Szepanowski

Germany

Patricia Szot

United States of America

Björn Tackenberg

Germany

Kanda Takashi

Japan

Shao-Jun Tang

United States of America

Malu Tansey

United States of America

Maki Tateyama

Japan

Juliet Taylor

Australia

Elena Tchougounova

Sweden

Irmgard Tegeder

Germany

Peter Teismann

United Kingdom

Andrea Tenner

United States of America

Niccolo Terrando

United States of America

Charlotte Teunissen

Netherlands

Dietmar Thal

Germany

Praveen Thumbikat

United States of America

Leonardo Tonelli

United States of America

Lars Tonges

Germany

Terrence Town

United States of America

Corinna Trebst

Germany
Marie-Eve Tremblay

Canada

James Tsai

United States of America

Kuen-Jer Tsai

Taiwan Republic of China

Styliani Tsirka

United States of America

Alexander Tsiskaridze

Georgia

Erdem Tuzun

Turkey

Neetu Tyagi

United States of America

Suresh Tyagi

United States of America

Kenneth Tyler

United States of America

William Tyor

United States of America

Marta Valcarcel-Ares

United States of America

Luc Vallieres

Canada

Bart Van Berckel

Netherlands

Anne-Marie Van Dam

Netherlands

Linda Van Eldik

United States of America

Barbara Van Leeuwen

Netherlands

E. Van Vliet

Netherlands

Pamela Vandevord

United States of America

Evros Vassiliou

United States of America

Elisabetta Vegeto

Italy

Claudia Verderio

Italy

Annamaria Vezzani

Italy 
Carl-Wilhelm Voge;

United States of America

Zvi Vogel

Israel

Christina Vogelaar
Germany

Cinzia Volonte

Italy

Bruce Volpe

United States of America

Joseph Volpe

United States of America

Hans-Christian Von Budingen

United States of America

Lucy Vulchanova

United States of America

Sheela Vyas

France

Christian Waeber

Ireland

David Wagner

United States of America

Amy Wagner

United States of America

Adam Walker

Australia

Rohan Walker

Australia

Zhe Wang

China

Guanghu Wang

United States of America

Xiaoyang Wang

Sweden

$X$ Wang

China

Clemens Warnke

Germany

James Washek

United States of America

John Watt

United States of America

Jyoti Watters

United States of America
Christine Webber

Canada

Sven Wehner

Germany

Jonathan Weinstein

United States of America

Martha Welch

United States of America

Han-Rong Weng

United States of America

Gary Wenk

United States of America

Andrew West

United States of America

Fletcher White

United States of America

Scott Whittemore

United States of America

Marlene Wiart

France

Heinz Wiendl

Germany

Donna M Wilcock

United States of America

Brigitte Wildemann

Germany

James Wiley

Australia

Jennifer Wilkinson-Berka

Australia

Kenneth Williams

United States of America

Didier Wion

France

Thomas Wisniewski

United States of America

Martin Witt

Germany

Yl Wu

Taiwan Republic of China

Heping Xu

United Kingdom

Fu-Shan Xue

China
Koji Yamanaka

Japan

Chul-Su Yang

Republic of Korea

Shu Yang

United States of America

Qing-Wu Yang

China

Honghong Yao

China

Elke Ydens

Belgium

Midori Yenari

United States of America

Gelareh Zadeh

Canada

Nahla Zaghloul

United States of America

Guang-Xian Zhang

United States of America

Ji Zhang

Canada

Jun-Ming Zhang

United States of America

Yumin Zhang

United States of America

Zheng Zhang

United States of America

Xuechu Zhen

China

Wendy Ziai

United States of America

Gregory Zipfel

United States of America

Berislav Zlokovic

United States of America

Robert Zorec

Slovenia

Zhiyi Zuo

United States of America

Author details

Department of Geriatrics, University of Arkansas

for Medical Sciences, Little Rock, Arkansas, USA.

${ }^{2}$ Department of Pathology, University of Toledo Health Sciences Campus, Toledo, USA. 